different lesion types. In initial analyses, we have explored the expression of beta 3 integrin and certain other adhesion molecules.

We demonstrate that the mRNA of beta 3 integrin is highly expressed in tumourgenic malignant melanomas and low or absent in compound nevi, paralleling these findings at the protein level ${ }^{1}$. We also demonstrate that the tetraspanin adhesion molecule CD9 is highly expressed in nevi with a low signal intensity detected in the melanoma sample. CD9 is a known marker whose function in melanomas is thought based on limited studies to inhibit metastatic potential.

To confirm the mRNA expression data for these two markers as a proof of principle, we examined 20 tumourigenic malignant melanomas and 20 dermal nevi by immunohistochemistry to evaluate protein level expression of the respective markers. All of the melanomas showed expression of the integrin in more than $60 \%$ of lesion cells, whereas all of the nevi expressed the marker in less than $20 \%$ of their cells. Conversely, CD9 was expressed in more than $80 \%$ of cells in all but one nevus, and in less than $20 \%$ of cells in all of the melanomas. Our findings confirm the microarray expression profile for these two markers at the protein level, providing a proof of principle for use of expression arrays to evaluate expression profiles for interesting markers in RNA extracted from human tumours.

Van Belle, P.A. et al Hum. Pathol. (in press)

Van Rompaey, Luc

\section{Tel, a frequent target of leukemic translocations, induces cellular aggrega- tion and influences expression of extra- cellular matrix components}

\author{
L. Van Rompaey, W. Dou \& G. Grosveld
}

Department of Genetics, St. Jude Children's Research Hospital, M emphis, Tennessee, USA

Tel (ETV6) is an ETS transcription factor that is found fused to a variety of tyrosine kinases and transcription factors in lymphoid and myeloid leukemias and in fibrosarcoma and mesoblastic nephroma. It contains two functional domains, a pointed (PNT) protein interaction domain and a DNA binding domain (DBD).

Retroviral transduction of a wild-type Tel cDNA into a clonal subline of NIH3T3 fibroblasts resulted in a reproducible, striking morphological change: at confluency, the cells reorganized into a specific 'bridge-like' pattern over the entire surface of the culture dish, and started migrating, thereby leaving circular holes in the monolayer. Thereafter cellular cord formation became apparent. This morphological change is most reminiscent to that of endothelial cells that differentiate into tubes in vitro. This effect may relate to the role of Tel in angiogenesis: a Tel knockout study showed that early in development Tel is required for the maintenance of the cell layer surrounding the endothelial cells in the yolk sac. Electron microscopic examination of the fibroblastic cellular cords revealed that they did not contain a lumen. Retroviral transduction of endothelial cells did induce aggregation of the cells but not cellular cord or tube formation. We inhibited the Tel-induced morphological change in the fibroblasts by coating the culture dishes with high but not low concentrations of fibronectin and collagen IV. Transduction of the fibroblasts with deletion mutants or point mutants of Tel, or with MN1-Tel and VP16-Tel, showed that both the pointed domain and the DNA binding domain of Tel are required to induce the morphological change. MN1-Tel and VP16-Tel as well as the ETS transcription factors Fli1 and Ets1, both expressed in endothelial cells, did not result in this morphological change. These results strongly suggest that native Tel, and probably target genes of Tel are causally involved in the induction of this phenotypic change.

We therefore searched for differentially expressed genes in Tel- versus vector- transduced fibroblasts using DNA filter arrays and antibody-based techniques. Thus far, 4 differentially expressed genes have been found that might relate to the observed phenotype. Expression differences were found to be consistent (present in at least 3 independent experiments), but low (1.5- to 3-fold different). More putative Tel target genes that function in ECM-build-up, cell adhesion and proteolysis are under investigation.

Vetrie, David

\section{Development of a PCR product-based microarray system for expression profil- ing of rat systems}

\author{
David Vetrie, Clare East, Adam Butler, Liz Campbell \\ $\&$ Tom Freeman
}

Gene Expression Group, The Sanger Centre, Wellcome Trust Genome Campus, Hinxton, CB10 1SA, UK

Over the past five years, we have been investigating means of performing highthroughput gene expression studies. Previously, we have developed an RT-PCRbased analysis system to monitor gene expression of a large collection of mouse genes (see The Jackson Laboratory, Gene Expression Database, dataset ID $\mathrm{J}: 46439)$. More recently, we have begun to work on the rat and investigate the use of high-density microarrays for performing large-scale expression studies. To this end, we are examining the potential of creating microarrays of RT-PCR products for all known rat genes. We are currently optimising the conditions necessary for arraying these PCR products on glass slides with a variety of surface chemistries using a first-generation arrayer from Genetic MicroSystems (GMS 417), as well as determining how these arrayed target DNAs perform during hybridisation. To further determine the sensitivity and reliability of this approach, we are preparing a 96-sample "mini"-microarray of rat genes for which the preliminary expression patterns have already been determined by RT-PCR. As we already have working RT-PCR assays for over 1,200 rat genes, we will have the capacity to generate gene arrays and RT-PCR expression profiles for a large collection of rat genes once the initial optimisation studies are completed. It will therefore be possible to directly compare microarrays and RT-PCR, and evaluate extensively their respective uses in large-scale expression studies. It is our intention to use these two technologies, together with other approaches for expression profiling, to investigate gene expression patterns across a range of normal rat tissues. Additionally, these technologies can be used to identify novel drug targets and help in understanding the mechanisms of action of therapeutic agents.

Vilo, Jaak

\section{Discovery of putative transcription factor binding sites from microarray-based gene expression profiles}

\author{
Jaak Vilo', Alvis Brazma', Inge Jonassen² \& Esko Ukkonen ${ }^{3}$ \\ ${ }^{1}$ European Bioinformatics Institute, EM BL O utstation-Hinxton, Cambridge, UK \\ ${ }^{2} U$ niversity of Bergen \\ ${ }^{3}$ University of Helsinki, Helsinki, Finland
}

We have analyzed gene upstream regions in the complete yeast genome for occurrences of regular expression type patterns with the goal to identify potential regulatory elements. First we clustered the upstream regions of all the genes by similarity of their expression profiles based on publicly available gene expression data by various methods. Next we developed a new sequence pattern discovery algorithm that searches exhaustively for a priori unknown patterns that are over- 